\title{
GENERALIZED THEOREMS OF DESARGUES FOR $n$-DIMENSIONAL PROJECTIVE SPACE
}

\author{
P. O. BELL
}

The purpose of the present note is to generalize to $n$ dimensions the celebrated two-triangle theorem of Desargues and its converse. ${ }^{1}$ The generalized theorems can be stated in simple forms which, nevertheless, suffice for each of the large number of special cases of the configurations involved. The terms concurrent and collinear will be used in the sense that $n$ lines are concurrent if there is at least one point lying on all $n$ of them, three points are collinear if there is at least one line containing them. The word simplex will denote an $n$-tuple of linearly independent points.

Two simplexes $A_{1} A_{2} \cdots A_{n}, A_{1}^{\prime} A_{2}^{\prime} \cdots A_{n}^{\prime}$ will be said to be in perspective from $\phi$ if there exists a point $\phi$ such that $\phi A_{1} A_{1}^{\prime}$, $\phi A_{2} A_{2}^{\prime}, \cdots$, and $\phi A_{n} A_{n}^{\prime}$ are each collinear triads. Each simplex determines a linear space of $n-1$ dimensions. Let $k$ points of one simplex coincide with the corresponding points of the other simplex $(k \geqq 0)$. Without loss of generality, the simplexes may be named in a manner such that $A_{i}$ coincides with $A_{i}^{\prime}$ if $i \leqq k$. With these definitions a generalization of Desargues' theorem is

Theorem 1. If two simplexes $A_{1}, A_{2} \cdots A_{n}, A_{1}^{\prime} A_{2}^{\prime} \cdots A_{n}^{\prime}$ are in perspective from a point $\phi$, and if $A_{j}$ does not coincide with $A_{j}^{\prime}$ for $k<j \leqq n$, there exists a point $P_{i j}$ common to $A_{i} A_{j}, A_{i}^{\prime} A_{j}^{\prime} \quad(k<i<j$, $i=1,2, \cdots, n-1)$ which is linearly dependent upon the $j-i$ points $P_{i}, P_{i+1}, \cdots, P_{j-1}$, in which $P_{h}$ denotes the point $P_{h} h+1$.

For $n=3$, the above stated theorem is obviously the theorem of Desargues.

For the proof of the theorem two cases are to be considered: Case (1). The point $\phi$ is distinct from each vertex of at least one of the simplexes. Case (2). The point $\phi$ coincides with a vertex of each simplex.

Proof. Case (1). Let $\phi$ be assumed to be distinct from each of the vertices $A_{1}, A_{2}, \cdots, A_{n}$. Let a fixed coordinate system be postulated in the join ${ }^{2}$ of the linear spaces determined by the simplexes. The

Received by the editors June 22, 1954 and, in revised form, January 6, 1955.

1 A statement of Desargues' theorem and of its converse may be found, for example, in Hodge and Pedoe, Methods of algebraic geometry, Cambridge University Press, 1947, p. 192.

2 The join of two linear spaces is the smallest linear space which contains the two spaces. 
property that the simplexes are in perspective from $\phi$ is that corresponding homogeneous coordinates of $\phi$ and the points of the simplexes are related by a system of linear equations which, on choosing multipliers of the points $A_{i}^{\prime}$ properly, assume the forms

$$
\begin{aligned}
& A_{1}^{\prime}=A_{1}, \quad A_{2}^{\prime}=A_{2}, \cdots, A_{k}^{\prime}=A_{k}, \\
& A_{i}^{\prime}=\phi+\lambda_{i} A_{i}
\end{aligned}
$$

The points $P_{k+1}, P_{k+2}, \cdots, P_{n-1}$ defined by

$$
P_{i}=A_{i}^{\prime}-A_{i+1}^{\prime}=\lambda_{i} A_{i}-\lambda_{i+1} A_{i+2} \quad(k<i<n)
$$

are points common to the corresponding pairs of lines $A_{i} A_{i+1}, A_{i}^{\prime} A_{i+1}^{\prime}$. Moreover, a point $P_{i j}$ common to the pair of lines $A_{i} A_{j}, A_{i}^{\prime} A_{j}^{\prime}$ is given by

$$
P_{i j}=A_{i}^{\prime}-A_{j}^{\prime}=\lambda_{i} A_{i}-\lambda_{j} A_{j} \quad(k<i<j<n) .
$$

In view of the expressions for the points $P_{i}(k<i<n)$ it follows immediately that

$$
P_{i j}=P_{i}+P_{i+1}+\cdots+P_{j-1} \quad(k<i<j \leqq n)
$$

In particular,

$$
P_{k+1 n}=P_{k+1}+P_{k+2}+\cdots+P_{n-1} .
$$

This completes the proof.

For the case of the theorem in which $k=0$, it is significant that the point $P_{i j}$ common to the lines $A_{i} A_{j}, A_{i}^{\prime} A_{j}^{\prime}$ lies in a linear space of $j-i-1$ dimensions which is a subspace of the linear space $S_{n-2}$ determined by the points $P_{1}, P_{2}, \cdots, P_{n-1}{ }^{3}$

In case $k \neq 0, A_{i}$ coincides with $A_{i}^{\prime}(i=1,2, \cdots, k)$. The points $P_{i j}(i \leqq k)$ may therefore be defined by the relations

$$
P_{i j}=P_{i}=A_{i} \quad(i=1,2, \cdots k, j \neq i) .
$$

Since the points $P_{i j}$ for $i>k$ satisfy the relations (1), any point $P_{i j}$ whatever lies in the linear space of $j-i-1$ dimensions determined by the points $P_{i}, P_{i+1}, \cdots, P_{j-1}$, each point $P_{i j}$ being either the point $P_{i}$ or a linear combination of these $j-i$ points.

Proof. Case (2). The point $\phi$ coincides with a vertex of each simplex. Let the vertices which coincide with $\phi$ be $A_{l}$ and $A_{m}{ }^{\prime}$. The sub-case in which $l=m$ can be disposed of as follows: Since $k$ denotes, as in Case (1), the number of points $A_{i}$ which coincide with their cor-

${ }^{3}$ If the simplexes $A_{1} A_{2} \cdots A_{n}, A_{1}^{\prime} A_{2}^{\prime} \cdots A_{n}^{\prime}$ are in distinct linear spaces $S_{n-1}, S_{n-1}^{\prime}$ it is, of course, obvious that the $P$ 's lie in the intersection space $S_{n-2}$. 
respondents $A_{i}^{\prime}$, the index $l$ will be one of the numbers $1,2, \cdots, k$. Without loss of generality, let the points $A_{1}, A_{l}$, and $A_{1}^{\prime}, A_{l}^{\prime}$ be renamed by permuting their indices so that the vertices which coincide with $\phi$ are said to be the points $A_{1}, A_{1}^{\prime}$. Therefore, with properly selected multipliers for $A_{1}$ and $A_{1}^{\prime}, A_{1}=A_{1}^{\prime}=\phi$. Moreover, as in Case (1) of the theorem, $A_{h}$ coincides with $A_{h}^{\prime}$ if $h \leqq k$. In this case $A_{h}$ is the common center of two sets of lines $A_{h} A_{j}, A_{h}^{\prime} A_{j}^{\prime}(j \neq h)$. Therefore, the points $P_{h j}(h \leqq k)$ may be defined as follows:

$$
P_{h j}=A_{h}=P_{h} \quad(h \leqq k, j>h) .
$$

Since $A_{i}, A_{i}^{\prime}$ are collinear with $\phi$, when $A_{i}$ does not coincide with $A_{i}^{\prime}$, they satisfy linear relations which, on choosing multipliers of the points $A_{i}^{\prime}$ properly, assume the forms

$$
A_{i}^{\prime}=\phi+\lambda_{i} A_{i}
$$

The points $P_{k+1}, P_{k+2}, \cdots, P_{n-1}$ are therefore defined by

$$
P_{i}=A_{i}^{\prime}-A_{i+1}^{\prime}=\lambda_{i} A_{i}-\lambda_{i+1} A_{i+1} \quad(k<i<n) .
$$

It follows that points common to pairs of lines $A_{i} A_{j}, A_{i}^{\prime} A_{j}^{\prime}$ are defined by

$$
P_{i j}=A_{i}^{\prime}-A_{j}^{\prime}=\lambda_{i} A_{i}-\lambda_{j} A_{j} \quad(k<i<j<n) .
$$

In view of (2), (3), and (4), the following relations

$$
\begin{array}{lr}
P_{i j}=P_{i}+P_{i+1}+\cdots+P_{j-1} & (k<i<j<n), \\
P_{h j}=P_{h} & (h \leqq k, j>h)
\end{array}
$$

hold. The proof of the theorem in this sub-case is complete.

For the proof of the remaining sub-case of the theorem, in which $A_{l}$ coincides with $A_{m}^{\prime}$ in $\phi(l \neq m)$, it is evident at once that $l$ and $m$ must both be greater than $k$ since $A_{i}=A_{i}^{\prime}(i \leqq k)$. (The distinguishing feature of this case is that the points of the simplexes which coincide in $\phi$ are not corresponding elements in the perspectivity.) Without loss of generality the names of the points $A_{l}, A_{k+1}$ and those of the points $A_{m}^{\prime}, A_{k+2}^{\prime}$ may be interchanged, so that the point $A_{k+1}$ will be said to coincide with the point $A_{\mathbf{k}+2}^{\prime}$ in $\phi$.

As in the sub-case of the theorem proved above, points $P_{h j}(h \leqq k)$ may be defined by equations (2).

Moreover, since on selecting multipliers of $A_{k+1}$ and $A_{k+2}^{\prime}$ properly, the relations $A_{k+1}=A_{k+2}^{\prime}=\phi$ hold, it follows from the hypothesis that with a proper selection of multipliers of $A_{i}^{\prime}$, we have

$$
A_{i}^{\prime}=\phi+\lambda_{i} A_{i}, \quad \lambda_{k+2}=0 \quad(i>k, i \neq k+1) .
$$


The points $P_{i j}$ may, therefore, be defined by the relations

$$
P_{i j}=A_{i}^{\prime}-A_{j}^{\prime}=\lambda_{i} A_{i}-\lambda_{j} A_{j} \quad(i, j>k, i \neq j) .
$$

On making use of the definitions of the points $P_{h}(h>k)$ it follows that

$$
\begin{gathered}
P_{k+1} j=\lambda_{k+1} A_{k+1}=P_{k+1} \quad(j>k+1), \\
P_{k+2}=-\lambda_{k+3} A_{k+3}, P_{i j}=P_{i}+P_{i+1}+\cdots+P_{j-1}(k+1<i<j) .
\end{gathered}
$$

The proof of the theorem is now complete.

Involved in the conclusion of Theorem 1 there are $(n-k-2)$ $\cdot(n-k-3) / 2$ nontrivial linear relations of the form

$$
P_{i j}=P_{i}+P_{i+1}+\cdots+P_{j-1} \quad(k<i<j-1 \leqq n-1) .
$$

The direct converse of Theorem 1 would postulate that among the points $P_{i j}$ common to the lines $A_{i} A_{j}, A_{i}^{\prime} A_{j}^{\prime}$ all of these linear relations are satisfied. It is interesting to find, however, that if any particular one of these relations holds, all of them will hold. Consequently, a generalization of Desargues' converse theorem appears in the following form:

Theorem 2. Let two simplexes $A_{1} A_{2} \cdots A_{n}, A_{1}^{\prime} A_{2}^{\prime} \cdots A_{n}^{\prime}$ be such that for each pair of integers $i, j(i \neq j ; i, j=1,2, \cdots, n)$ there exists a corresponding point $P_{i j}$ common to the lines $A_{i} A_{j}, A_{i}^{\prime} A_{j}^{\prime}$. Let $k$ denote the number of points of the simplex $A_{1} A_{2} \cdots A_{n}$ which coincide with the corresponding points of the simplex $A_{1}^{\prime} A_{2}^{\prime} \cdots A_{n}^{\prime}$ $(n \geqq k \geqq 0)$. If $k \geqq n-2$, the simplexes are in perspective from a point. If $k<n-2$, let the points of the simplexes be so named that $A_{i}$ coincides with $A_{i}^{\prime}(i=1,2, \cdots, k)$. If a particular point $P_{r s}(k<r<s-1 \leqq n-1)$ is linearly dependent upon all of the $s-r$ points $P_{r}, P_{r+1}, \cdots, P_{s-1}$, the simplexes are in perspective from a point.

If $k=n$, the simplexes coincide. They are therefore in perspective from an arbitrary point $\phi$.

If $k=n-1$, the simplexes are in perspective from any point $\phi$ collinear with $A_{n}, A_{n}^{\prime}$.

If $k=n-2$, according to hypothesis a point $P_{n-1}$ common to the lines $A_{n-1} A_{n}, A_{n-1}^{\prime} A_{n}^{\prime}$ exists. The lines $A_{n-1} A_{n-1}^{\prime}, A_{n} A_{n}^{\prime}$ are, therefore, in a common plane and consequently concur in a point $\phi$ from which the simplexes are in the perspective.

If $k<n-2$, let multipliers of the linearly dependent points $P_{r s}, P_{r}$, $P_{r+1}, \cdots, P_{s-1}$ be selected such that the linear relation assumes the form 


$$
P_{r}+P_{r+1}+\cdots+P_{s-1}+P_{s r}=0, \quad P_{r s}=-P_{s r} .
$$

The points are themselves defined by linear relations

$$
\begin{aligned}
& P_{r s}=\alpha_{r s} A_{r}+\beta_{r s} A_{s}=\alpha_{r}^{\prime} A_{r}^{\prime}+\beta_{r s}^{\prime} A_{! s}^{\prime} \\
& P_{i}=\alpha_{i} A_{i}+\beta_{i+1} A_{i+1}=\alpha_{i}^{\prime} A_{i}^{\prime}+\beta_{i+1}^{\prime} A_{i+1}^{\prime} \\
& \quad(i=r, r+1, \cdots, s-1) .
\end{aligned}
$$

In view of the linear independence of the $A$ 's and of the $A$ "s, the coefficients of the $A$ 's and of the $A^{\prime \prime}$ 's which result from substituting the above relations into (6) must be equated to zero. The results follow:

$$
\begin{aligned}
& \alpha_{r}-\alpha_{r s}=\beta_{s}-\beta_{r s}=\alpha_{i}+\beta_{i}=0, \\
& \alpha_{r}^{\prime}-\alpha_{r s}^{\prime}=\beta_{s}^{\prime}-\beta_{r s}^{\prime}=\alpha_{i}^{\prime}+\beta_{i}^{\prime}=0 \quad(i=r+1, \cdots, s-1) .
\end{aligned}
$$

The relations (7) together with (8) imply that

$$
\alpha_{r} A_{r}-\alpha_{r}^{\prime} A_{r}^{\prime}=\alpha_{r+1} A_{r+1}-\alpha_{r+1}^{\prime} A_{r+1}^{\prime}=\cdots=\beta_{s}^{\prime} A_{s}^{\prime}-\beta_{s} A_{\text {s. }}
$$

A point $\phi$ is thus defined which is a point common to each of the lines $A_{r} A_{r}^{\prime}, A_{r+1} A_{r+1}^{\prime}, \cdots, A_{8} A_{?}^{\prime}$.

By means of the following argument the point $\phi$ will be shown to be a point common to each of the lines $A_{s+1} A_{s+1}^{\prime}, \cdots, A_{n} A_{n}^{\prime}$. Let $p$ denote any one of the integers $s+1, s+2, \cdots, n$. According to the hypothesis, points $P_{p r}, P_{p r+1}, \cdots, P_{p s}$ exist which are common to the respective pairs of lines $\left(A_{p} A_{r}, A_{p}^{\prime} A_{r}^{\prime}\right),\left(A_{p} A_{r+1}, A_{p}^{\prime} A_{r+1}^{\prime}\right), \cdots$, $\left(A_{p} A_{s}, A_{p}^{\prime} A_{s}^{\prime}\right)$. These points are, therefore, defined by such linear forms as the following

$$
\begin{aligned}
P_{p r} & =a_{p r} A_{p}+b_{p r} A_{r}=a_{p r}^{\prime} A_{p}^{\prime}+b_{p r}^{\prime} A_{r}^{\prime}, \\
P_{p r+1} & =a_{p r+1} A_{p}+b_{p r+1} A_{r+1}=a_{p r+1}^{\prime} A_{p}^{\prime}+b_{p r+1}^{\prime} A_{r+1}^{\prime}, \\
& \cdot \cdot \cdot \cdot \cdot \cdot \cdot \cdot \cdot \cdot \cdot \cdot, \\
P_{p s} & =a_{p s} A_{p}+b_{p s} A_{\imath}=a_{p s}^{\prime} A_{p}^{\prime}+b_{p s}^{\prime} A_{s .}^{\prime}
\end{aligned}
$$

From these relations the following ones are deduced:

$$
\begin{aligned}
& a_{p r} A_{p}-a_{p r}^{\prime} A_{p}^{\prime}=b_{p r}^{\prime} A_{r}^{\prime}-b_{p r} A_{r}, \\
& a_{p r+1} A_{p}-a_{p r+1}^{\prime} A_{p}^{\prime}=b_{p r+1}^{\prime} A_{r+1}^{\prime}-b_{p r+1} A_{r+1} \text {, }
\end{aligned}
$$

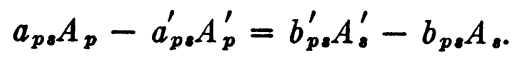


It follows that the line $A_{p} A_{p}^{\prime}$ intersects each of the lines $A_{r} A_{r}^{\prime}$, $A_{r+1} A_{r+1}^{\prime}, \cdots, A_{s} A_{s}^{\prime}$. If $A_{p} A_{p}^{\prime}$ should coincide with one of these lines, it could not coincide with another, because of the linear independence of the points of the simplexes. Therefore in such a case $A_{p} A_{p}^{\prime}$ contains the point $\phi$. If $A_{p} A_{p}^{\prime}$ does not coincide with any of these lines, it must, nevertheless, contain the point $\phi$. For if $A_{p} A_{p}^{\prime}$ does not contain $\phi$, its points of intersection with the above named lines and $\phi$ are in a common plane. But this latter condition cannot hold because the lines $A_{p} A_{p}^{\prime}, A_{r} A_{r}^{\prime}, A_{r+1} A_{r+1}^{\prime}, \cdots, A_{s} A_{s}^{\prime}$ would then be in a common plane and the linear independence of the points of each simplex would be violated. The proof of the theorem is now complete.

Various corollaries can be stated, among which the following ones are noteworthy:

Corollary 1. Let two simplexes $A_{1} A_{2} \cdots A_{n}, A_{1}^{\prime} A_{2}^{\prime} \cdots A_{n}^{\prime}$ be defined in a manner such that corresponding to each pair $(i, j)$ a point $P_{i j}$ common to the lines $A_{i} A_{j}, A_{i}^{\prime} A_{j}^{\prime}$ exists. If there exists a triad $(r, s, t)$ such that (a) $A_{r}, A_{s}, A_{t}$ do not coincide with the corresponding points $A_{r}^{\prime}, A_{s}^{\prime}, A_{t}^{\prime}$, and (b) $P_{r t}$ is collinear with $P_{r s}$ and $P_{s t}$, the simplexes are in perspective from a point.

Corollary 2. Let two simplexes $A_{1} A_{2} \cdots A_{n}, A_{1}^{\prime} A_{2}^{\prime} \cdots A_{n}^{\prime}$ and points $P_{i j}(i \neq j, i=1,2, \cdots n)$ be defined as in Corollary 1. Let $P_{i}$ denote the points $P_{i+1}(i=1,2, \cdots, n-1)$. If the point $P_{l n}$ is linearly dependent upon the $n-1$ points $P_{1}, P_{2}, \cdots, P_{n-1}$ but not upon a smaller number of these points, the simplexes are in perspective from a point.

The following theorem provides a geometric interpretation relative to the simplexes of a linear relation among the points $P_{1}, P_{2}, \cdots$, $P_{n-1}$.

Theorem 3. Let two simplexes $A_{1} A_{2} \cdots A_{n}, A_{1}^{\prime} A_{2}^{\prime} \cdots A_{n}^{\prime}$ be defined in a manner such that corresponding to each pair $(i, j)$ a point $P_{i j}$ common to the lines $A_{i} A_{j}, A_{i}^{\prime} A_{j}^{\prime}$ exists. Let $P_{i}$ denote the point $P_{i}{ }_{i+1}$. If a linear relation exists among the $n-1$ points $P_{1} P_{2}, \cdots P_{n-1}$, but no such relation exists among a smaller number of these points, corresponding points $A_{i}, A_{i}^{\prime}(i \neq 1, n)$ of the two simplexes coincide, and the simplexes are in perspective from a point.

The case of Theorem 1 which corresponds to $k=0$ may be generalized in another direction as follows:

Theorem 4. If two simplexes $A_{1} A_{2} \cdots A_{n}, A_{1}^{\prime} A_{2}^{\prime} \cdots A_{n}^{\prime}$ are in 
perspective from $a$ point and if each point $A_{i}$ is distinct from the corresponding point $A_{i}^{\prime}$, corresponding to each pair $(i, j)(i \neq j$, $i=1,2, \cdots, n)$ there exists a point $P_{i j}$ common to $A_{i} A_{j}, A_{i}^{\prime} A_{j}^{\prime}$ such that the points of each set $P_{l_{j_{l}}}, P_{1 j_{1}}, \cdots P_{n j_{n}}$, in which $j_{1} j_{2}, \cdots, j_{n}$ is a permutation of the set of integers $1,2, \cdots, n$, are linearly dependent.

In a similar manner, the following general theorem incorporates, as special cases, Theorem 3 and Corollary 2 of Theorem 2:

Theorem 5. If two simplexes $A_{1} A_{2} \cdots A_{n}, A_{1}^{\prime} A_{2}^{\prime} \cdots A_{n}^{\prime}$ are such that corresponding to each pair $(i, j)(i \neq j, i=1,2, \cdots, n)$ there exists a point $P_{i j}$ common to $A_{i} A_{j}, A_{i}^{\prime} A_{j}^{\prime}$, and if either one of the following conditions holds, the simplexes are in perspective from a point: (a) among the $n$-points of any set $P_{1 j_{1}}, P_{2 j_{2}}, \cdots, P_{n j_{n}}$, in which $j_{1}, j_{2}, \cdots, j_{n}$ is a permutation of the set of integers $1,2, \cdots, n$ such that $i \neq j_{i}$ $(i=1,2, \cdots, n)$, but not among any subset of these points, there exists a linear dependence; (b) among $n-1$ points of such a set $P_{1 j_{1}}, P_{2 j_{2}}, \ldots$, $P_{n j_{n}}$, but not among a smaller number of these points, there exists a linear dependence. If (a) forms part of the hypothesis, $A_{j}$ does not coincide with $A_{j}^{\prime}$ for any value of $j$. If (b) forms part of the hypothesis and if $P_{h j_{h}}$ is the point not involved in the linear dependence, $A_{i}$ coincides with $A_{i}^{\prime}$ in each of the $n-2$ instances in which $i \neq h, j_{h}$.

Theorems 3, 4, and 5 may be proved, without serious difficulty, by methods similar to those already applied. Their proofs will therefore be omitted.

If in the hypothesis of Theorem 5 condition (b) is replaced by the condition that a linear dependence exists among $n-2$ points of a set $P_{1 j_{1}}, P_{2_{j_{2}}}, \cdots, P_{n j_{n}}$, the conditions are insufficient to force the simplexes to be in perspective from a point. To illustrate, let $P_{h}, P_{k}$ denote two points which do not appear in a given linear relation among the remaining $n-2$ points of the set $P_{1}, P_{2}, \cdots, P_{n}$. In view of the linear independence of the points of each simplex, it may be easily shown that such a relation places no restriction upon any of the points $A_{h}, A_{h+1}, A_{k}, A_{k+1}, A_{h}^{\prime}, A_{h+1}^{\prime}, A_{k}^{\prime}, A_{k+1}^{\prime}$. The existence of the points $P_{h}, P_{k}$ having already been assumed, the existence of points $\phi_{h}, \phi_{k}$ common to the pairs of lines $A_{h} A_{h}^{\prime}, A_{h+1} A_{h+1}^{\prime}$ and $A_{k} A_{k}^{\prime}$, $A_{k+1} A_{k+1}^{\prime}$, respectively, is assured, but the points $\phi_{h}, \phi_{k}$ do not necessarily coincide. Consequently, under these conditions the simplexes are not, in general, in perspective from a point.

UNIVERSITY OF KANSAS 